\title{
Symptomatic, Operable Tarlov Cysts: A Myth or a Reality?
}

\author{
Ajay Kothari, Vibhu Krishnan Viswanathan*, Ketan Shripad Khurjekar and Parag K Sancheti \\ Ganga Hospital, India
}

Submission: 5 June, 2016; Published: 28 June, 2016

*Corresponding author: Vibhu Krishnan Viswanathan, Ganga Hospital, 313, Mettupalayam Road, Saibaba Koil, Coimbatore, Tamil Nadu, 641043, India, Tel: 778819219; Email: drvibu007@gmail.com

\begin{abstract}
The presence of perineural cysts within the sacral spinal canal was initially described in the literature as early as 1938, when Tarlov had identified a similar lesion during one of his autopsies. While a majority of these lesions are asymptomatic and represent incidental findings observed on MRIs or CT scans, there have been described rare instances in the literature, when these lesions have resulted in symptoms which required surgical intervention. Coccygodynia, dyspareunia, perianal pain and cauda equina syndrome have been described as definitive indications for surgery. However, the role of surgery in relieving back pain or radicular symptoms following Tarlov cysts has remained controversial. The current article describes a similar lesion observed in one of our patients. The purpose of this report is to highlight the fact that tarlov cyst can definitely be a potential cause for back and leg symptoms in certain patients and surgical decompression in the form of complete cyst excision along with the walls may be considered a safe and effective option after failure of conservative trial.
\end{abstract}

Keywords: Sacral tarlov cyst; Back pain; Radicular pain; Laminectomy with excision

\section{Introduction}

The presence of perineural cysts within the sacral spinal canal was initially described in the literature as early as 1938 , when Tarlov had identified a similar lesion during one of his autopsies [1]. These rare cerebro-spinal fluid-filled lesions arising from the nerve root sheaths of the dorsal root ganglion, have thence, been described by various authors with diverse clinical and radiological presentations [2]. While a majority of these lesions are asymptomatic [3], there have been described instances in the literature, when these lesions have resulted in symptoms such as low back or radicular pains, coccygodynia or cauda equina syndrome [3]. Since the pathogenesis of these lesions remains ambiguous [2], the ideal mode of management of these symptomatic cysts has remained controversial and poorly understood.

\section{Case Report}

A 62 year old lady presented with low back pain, radiating to the posterior thighs and calves bilaterally, more on the right side. The pain had started over a period of two months, gradual in onset, progressive and claudicating in nature. Over the past 10 days, the pain had increasingly become more intense, resulting in recurrent night pains. Examination showed axial spinal tenderness at lower lumbar and sacral regions. Straight leg rising was normal bilaterally. There was mild blunting of sensations along S2 and S3 dermatomes on right side, while motor examination, superficial as well as deep tendon reflexes were grossly normal. Anal tone and anal reflexes were preserved. Clinical examination at this point of time revealed no relevant positive findings at bilateral hips, knees and sacro-iliac joints.

Roentgenograms of the lumbo-sacral spine and pelvis did not reveal pathology. The previous MRI scan was compared with a new post contrast MRI of THE Lumbosacral spine revealing multiple fluid filled cystic lesions around S2-3 levels (Figures 1 \& 2). Axial tomography revealed scalloping and erosions of the bone around S2-3, following compressions from posterior sacral cysts (Figure $3)$. The patient was given a trial of conservative management with adequate analgesia and extensive physiotherapy and rehabilitation for 6 weeks, which did not provide much relief. After an informed consent regarding the situation and the possible management options available, the patient opted for the surgical intervention. She was planned for sacral laminectomy and cyst excision, as described below. 


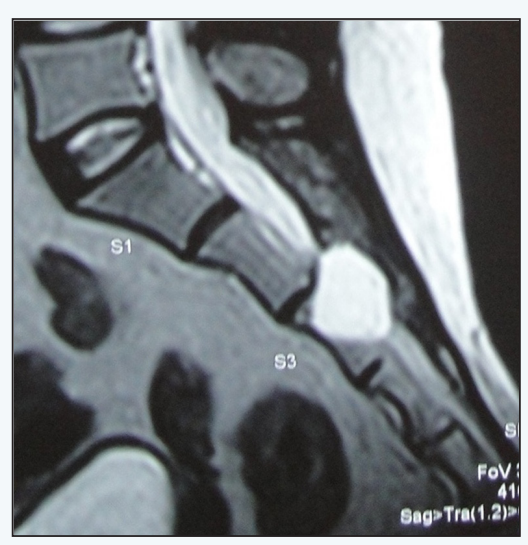

Figure 1: Sagittal MRI section showing Tarlov cyst at S2,3 levels

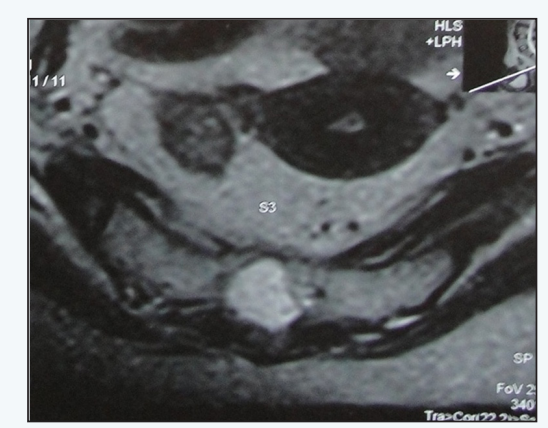

Figure 2: Axial MRI sections indicating the extent of the Tarlov cyst.

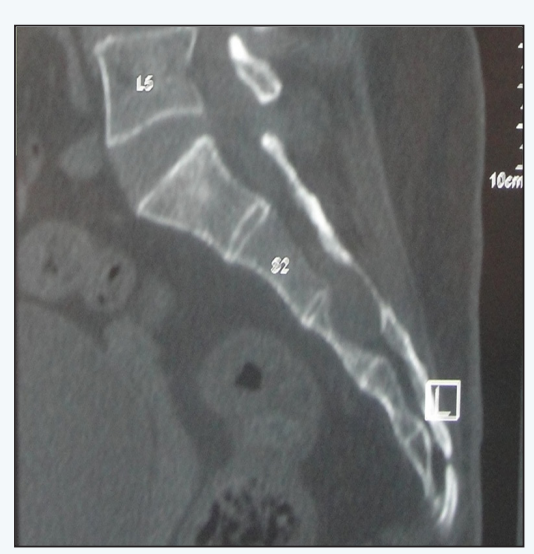

Figure 3: Sagittal CT scan showing scalloping of the posterior wall of sacrum, secondary to pressure from the growing Tarlov cyst.

\section{Surgical procedure}

Through midline posterior incision, the sacrum was exposed. Bilateral laminectomy was carried out at S2 and S3 levels. The cysts were identified under microscopic guidance and the margins carefully dissected out. There were four cysts in total, two of which could be excised entirely (Figure 4). There were two more cysts in close proximity to $\mathrm{S} 2,3$ roots, which were aspirated preserving the intact nerve sheath. The patient reported immediate postoperative relief of symptoms and had an uneventful recuperation after the surgery. Final histo-pathological examination of the cyst wall correlated with the clinical picture, thereby confirming the pre-operative diagnosis of a peri-neural cyst. During follow-up at 6 months, the patient had pain of 2/10 on VAS scale in the lower limbs and back; and could perform all her activities without any gross restrictions.

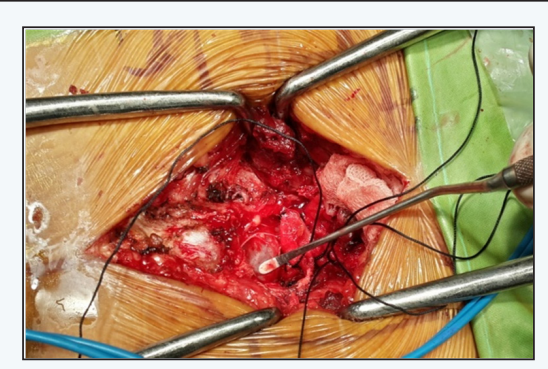

Figure 4: Intra-operative picture indicating the location of the sacral cyst.

\section{Discussion}

The terms 'Tarlov cysts', 'nerve root cysts', 'perineurial cysts' or 'perineural cysts' are synonymous and describe certain pathological formations, located in the space between the periand endoneurial linings of the spinal posterior nerve root sheath at the dorsal root ganglion [1,2]. These cysts typically occur on the extradural components of sacral nerve roots, and are multiple and multiloculated. They are known to be associated rarely with Marfan's syndrome, Ehlers-Danlos syndrome and Loeys-Dietz syndrome [2]. These cysts are classified presently as type 2 spinal meningeal cysts, under the classification system introduced by Nabors et al. [4,5]. The walls of these cysts contain nerve fascicles and histo-pathological examination reveals nerve fibres and ganglion cells [6].

Tarlov cysts can be significantly symptomatic at times, manifesting with local bony pains following bony erosions and insufficiency fractures or with radicular symptoms secondary to mechanical, adjacent nerve root compression, cauda equina syndrome, coccygodynia, perianal pain and dyspareunia $[7,8]$. One of the most convincing theories, regarding the persistent progression of size and symptoms in certain selective cystic lesions has been the "ball-valve theory" mechanism [9]. In those cysts, where a valve-like mechanism exists at the mouth of the lesion, restricting out-flow of the cerebrospinal fluid, the symptoms may continually progress as the fluid accumulates in the confined space under gravitational force. Those cysts with free bidirectional flow across the mouth may never manifest clinically [2].

Various hypotheses on the pathogenesis of formation of these cysts have been purposed in the literature $[1,9,10]$. These cysts are most probably acquired lesions [9], developing secondary to one of the following mechanisms: trauma resulting from the 
hydrostatic and pulsatile forces of cerebrospinal fluid in the involved spinal segments, hemorrhages into spinal nerve roots, degenerative processes involving posterior nerve roots, or migration of subarachnoid hemorrhage along the root. However, congenital arachnoidal proliferation was also described as the possible etiology by Fortuna et al. [11].

The indications to treat Tarlov cysts and the preferred line of management in symptomatic lesions remain controversial till date. Successful outcome of surgery depends upon a careful selection of patients. Cauda equina syndrome, coccygodynia, perianal pain and dyspareunia are four symptoms which are described to significantly improve following the surgical excision [12]. However, back and radicular pain are non-specific symptoms related to tarlov cysts. Voyadzis et al. [6] had proposed that cysts larger than $1.5 \mathrm{~cm}$ size with significant radicular symptoms had satisfactory relief following surgical decompression. Tanaka et al. [12] described a "positive filling defect" sign to identify symptomatic lesions. On CT myelography, Tarlov cysts which are valved proximally do not accumulate any dye. On the contrary, meningeal diverticula which allow free flow of CSF into and out of the lesions fill with dye. A positive filling defect was discussed as a definitive indication for surgery as such lesions tend to progressively accumulate fluid. A trial of conservative management always needs to be considered initially before surgical decompression in Tarlov's cysts and other treatable causes of back and radicular pains need to be excluded primarily.

The reported invasive treatment options available in the literature so far [3,5,7-10] include lumbar CSF drainage, lumbar peritoneal and cyst-subarachnoid shunt placements, CT-guided percutaneous aspirations of the cyst with or without infusion of fibrin glue, laminectomy or laminoplasty for decompression of the cyst, partial cyst removal and neck ligation with or without nerve root resection, partial cyst removal and cyst wall imbrication, microsurgical cyst removal and cyst wall imbrication (along with defect repairing with muscle, Gelfoam, or fibrin glue) and microsurgical fenestration of sacral perineural cysts to the thecal sac. Various complications have been reported to be associated with these interventions including prostatitis, cerebellar bleeding, CSF leak, dislodgement of muscle patch, urinary incontinence, recurrent pain, displacement of catheter and deep venous thrombosis [3,5,7-10]. In a retrospective analysis of 15 operated cases, Elsawaf et al. [13] observed complications in 2 patients (superficial wound infection and CSF leak) following surgical decompression of symptomatic tarlov cysts. Complete resolution of symptoms was observed in all these patients post-operatively.

\section{Conclusion}

In the present case, THE patient's symptoms persisted despite an adequate conservative trial. Therefore, surgical decompression was considered. 4 cysts were identified at the S2-3 levels. We performed sacral laminectomy, followed by complete excision of two cysts along with their walls, where the nerve roots were separable intra-operatively and aspirated the remaining two lesions, where such complete excision was not possible. All procedures were performed under microscopic guidance.

We have described a rare scenario of sacral Tarlov cyst causing axial back and radicular pain, which completely resolved following decompression and cyst excision. These lesions are rarely symptomatic and therefore the current evidence in the literature on operative management of Tarlov cysts is based on retrospective data and anecdotal evidence [3,5,7-10]. Large scale, prospective studies are needed to understand the prognostic implications of these lesions and identify the definitive surgical indications. Such studies may also help us to learn the ideal surgical approach to symptomatic cysts. If performed in the right candidate, surgical decompression is a safe and effective treatment modality for symptomatic tarlov cysts.

\section{Reference}

1. Tarlov IM (1938) Perineural cysts of the spinal nerve roots. Arch Neural Psychiatry 40(6): 1067-1074.

2. Chaiyabud P, Suwanpratheep K (2006) Symptomatic Tarlov cyst: Report and review. J Med Assoc Thai 89(7): 1047-1050.

3. Prashad B, Jain AK, Dhammi IK (2007) Tarlov cyst: Case report and review of literature. Indian J Orthop 41(4): 401-403.

4. Smith ZA, Li Z, Raphael D, Khoo LT (2011) Sacral laminoplasty and cystic fenestration in the treatment of symptomatic sacral perineurial (Tarlov) cysts: Technical case report. Surg Neurol Int 2: 129.

5. Nabors MW, Pait TG, Byrd EB, Karim NO, Davis DO, et al. (1988) Updated assessment and current classification of spinal meningeal cysts. J Neurosurg 68(3): 366-377.

6. Voyadzis JM, Bhargava P, Henderson FC (2001) Tarlov cysts: a study of 10 cases with review of the literature. J Neurosurg 95(1): 25-32.

7. Jain SK, Chopra S, Bagaria H, Mathur PP (2002) Sacral perineural cyst presenting as chronic perineal pain: a case report. Neurol India 50(4): 514-515.

8. Peh WC, Evans NS (1992) Tarlov cysts-another cause of sacral insufficiency fractures? Clin Radiol 46(5): 329-330.

9. Uemura K, Komatsu Y, Shibata T, Kobayashi E, Nose T (2001) Sacral meningeal cyst associated with valve-like mechanism - case report. Neurol Med Chir (Tokya) 41(5): 288-291.

10. Kunz U, Mauer UM, Waldbaur H (1999) Lumbosacral extradural arachnoid cysts: diagnosis and indications for surgery. Eur Spine J 8(3): 218-222.

11. Fortuna A, La Torre E, Ciappetta P (1977) Arachnoid diverticula : a unitary approach to spinal cysts communicating with the subarachnoid space. Acta Neurochir (Wien) 39(3-4): 259-268.

12. Tanaka M, Nakahara S, Ito Y, Nakanishi K, Sugimoto Y, et al. (2006) Surgical results of Sacral Perineural (Tarlov) cysts. Case Report. Acta Med Okayamaa 60(1): 65-70.

13. Elsawaf A, Awad TE, Fesal SS (2016) Surgical excision of symptomatic sacral perineurial cyst: case series and review of literature. Eur Spine J. 